\title{
OMISIÓN DE DAR RESPUESTA, BARRERAS BUROCRÁTICAS Y DEBILIDAD EN LA FISCALIZACIÓN COMO FACTORES DE VULNERACIÓN AL DERECHO DE PETICIÓN.
}

\author{
OMISSION TO ANSWER, BUROCRATIC BARRIERS AND WEAKNESS IN THE AUDIT AS FACTORS \\ OF VULNERATION TO THE RIGHT OF PETITION.
}

Isaias Samael Chambilla Aduviri ${ }^{1}$

Edward Percy Vargas Valderrama²

Aceptado: $17 / 09 / 2019$

Publicado online:15/01/2020

\section{RESUMEN}

La presente investigación tiene por objetivo fundamental, determinar cuáles son los principales factores que inciden en la vulneración al Derecho de Petición. Tacna, 2016. El trabajo corresponde a una investigación de tipo no experimental (ex post facto). Para tal propósito se consideró la información obtenida a través del cuestionario y la entrevista. La omisión de dar respuesta por escrito en el plazo legal y las barreras burocráticas ilegales por la entidad obligada, y la debilidad de las labores de fiscalización por las autoridades son los principales factores que inciden en la vulneración al Derecho de Petición. Tacna, 2016.

Palabras Clave: Vulneración al Derecho de Petición, plazo legal, derecho de petición.

\begin{abstract}
The objective of this investigation is to determine which are the main factors that affect the violation of the Petition Law. Tacna, 2016. The work corresponds to a non-experimental investigation (ex post facto). For this purpose, the information obtained through the questionnaire and the interview was considered. The omission of responding in writing in the legal term and illegal bureaucratic barriers by the obliged entity, and the weakness
\end{abstract}

\footnotetext{
${ }^{1}$ Magíster en Derecho Constitucional. Secretario judicial en la Corte Superior de Justicia de Tacna, Tacna, Perú

${ }^{2}$ Magíster en Derecho Constitucional, Jefe de la Oficina de Defensoría de Tacna, docente de la Facultad de Derecho y Ciencias Políticas de la Universidad Privada de Tacna, Tacna, Perú
} 
of the inspection tasks by the authorities are the main factors that affect the violation of the Petition Law. Tacna, 2016.

Keywords: Violation of Petition Law, legal term, right of petition.

\section{INTRODUCCIÓN}

El Derecho es dinámico, por cuanto en su trayecto prospectivo el estudio del Derecho de Petición adquiere gran relevancia, indubitablemente el mundo globalizado así lo exige, y la concretización del mismo se plasma en el ejercicio pleno de los ciudadanos en sus relaciones con la Administración Pública. La Petición es un derecho fundamental. Habida cuenta, del Derecho de Petición comporta un conjunto de obligaciones explícitas o implícitas para la autoridad, que son: a) facilitar los medios para que el ciudadano pueda ejercitar el derecho de petición sin trabas innecesarias; b) abstenerse de cualquier acto que pueda significar una sanción al peticionante c) exteriorizar el hecho de la recepción de la petición; d) dar el curso que corresponda a la petición; e) resolver la petición con la motivación correspondiente; y f) comunicar al peticionante lo que se hubiese resuelto con relación a su pedido. (STC N 09412001-AA/TC, fundamento. Salazar, R. (1999) afirma que el núcleo del Derecho de Petición en el Perú implica, por un lado, el ejercicio individual o colectivo del derecho, con el límite de que los miembros de las Fuerzas Armadas y Policiales sólo pueden ejercerlo individualmente; y, por otro, la obligación de la autoridad competente de dar al interesado una respuesta dentro del plazo legal, bajo responsabilidad. Chacón, M. (2015), en el estudio “El Derecho de Petición y el Silencio", concluye la importancia que tiene el Derecho de Petición, donde la respuesta también debe ser oportuna, es decir que debe dictarse dentro del plazo o término señalado por la ley. Gamboa, Y. (2014), refiere que el modelo del Estado Social de Derecho, Colombia garantiza la aplicación de los derechos fundamentales, siendo uno de estos el Derecho de Petición. No todas las peticiones dirigidas a los poderes políticos se realizan en el ejercicio del Derecho de Petición, Bartra (2012). En nuestro país desde el inicio de su vida Republicana, el Derecho de Petición siempre ha estado presente en cada una de nuestras constituciones a lo largo de la historia, hasta hoy; teniendo en cuenta que existen importantes diferencias desde su establecimiento por primera vez, sobre todo en relación al tema de los efectos jurídicos que produce como resultado de su aplicación y también del llamado silencio administrativo. Al respecto, Salazar (1999) manifiesta que, para determinar la naturaleza del Derecho de Petición en el Perú, el método que nos parece más apropiado es el de relacionarlo con los demás derechos consagrados en la Constitución Política, visión sistemática ésta que nos permitirá captar su esencia jurídica. La Defensoría del Pueblo en ese sentido, promociona la recuperación de los valores éticos como la responsabilidad en el ejercicio de la función pública, la transparencia, el buen gobierno y el buen servicio al ciudadano, que constituyen la base de la institucionalidad democrática en todos los niveles de gobierno en el que se desarrollen. (Informe Defensorial № 133, pp.13-14). Díaz Mendoza (2013) indica lo que sí podría realizar la Defensoría del Pueblo, al recibir una queja por presunta vulneración del derecho constitucional.

\section{OBJETIVOS}

a) Establecer en qué medida la entidad obligada omite dar respuesta por escrito en el plazo legal en los casos presentados para la petición.

b) Establecer en qué medida se dan las barreras burocráticas ilegales en los casos de petición. 
c) Establecer en qué medida existe debilidad de las labores de fiscalización en los casos de petición.

d) Establecer en qué medida existe vulneración al Derecho de Petición en los casos presentados.

\section{METOdOLOGÍA}

Investigación socio-jurídica no experimental (ex post facto) descriptivo y explicativo. Retrospectiva en el análisis documental y prospectivo en la parte de apreciaciones de los implicados en la muestra. El estudio comprende los casos por vulneración al Derecho de Petición, vistos por la Oficina de la Defensoría del Pueblo en la ciudad de Tacna, período 2016. La muestra aleatoria estuvo compuesta por 14 Magistrados, 50 Abogados y 30 Docentes. Se realizó búsqueda en Registro documental, sistematización de expedientes y la entrevista personalizada. Los instrumentos de medición han sido validados mediante juicio de expertos (especialistas en el tema y en metodología de la investigación).

\section{RESULTADOS}

A fin de establecer los principales factores que están incidiendo en la vulneración al Derecho de Petición. Tacna, 2016; se aplicaron tres instrumentos de medición: el cuestionario aplicado a los profesionales en Derecho; la cédula de entrevista a los Magistrados y la ficha de análisis a los expedientes. El resultado de la aplicación a abogados se muestra en la tabla 01

\section{Tabla 1}

\section{Cuestionario a abogados}

\begin{tabular}{|c|c|c|c|c|c|c|}
\hline \multirow{3}{*}{$\begin{array}{l}\text { Conocimiento sobre la petición comporta un conjunto de } \\
\text { obligaciones explícitas o implícitas para la autoridad, en el } \\
\text { que se incluye el derecho a obtener una respuesta por } \\
\text { escrito dentro del plazo legal, bajo responsabilidad. }\end{array}$} & \multicolumn{6}{|c|}{ Muestra } \\
\hline & \multicolumn{2}{|c|}{ Magistrados } & \multicolumn{2}{|c|}{ Abogados } & \multicolumn{2}{|c|}{ Docentes } \\
\hline & $\mathrm{N}^{\circ}$ & $\%$ & $\mathrm{~N}^{\circ}$ & $\%$ & $\mathrm{~N}^{\circ}$ & $\%$ \\
\hline SI & 14 & 100 & 45 & 90 & 27 & 90 \\
\hline NO & 0 & 0 & 5 & 10 & 3 & 10 \\
\hline TOTAL & 14 & 100 & 50 & 100 & 30 & 100 \\
\hline \multirow{3}{*}{$\begin{array}{l}\text { En la mayoría de los casos de petición existe apatía de las } \\
\text { autoridades al resolver los pedidos sin observar el plazo } \\
\text { legal. }\end{array}$} & \multicolumn{6}{|c|}{ Muestra } \\
\hline & \multicolumn{2}{|c|}{ Magistrados } & \multicolumn{2}{|c|}{ Abogados } & \multicolumn{2}{|c|}{ Docentes } \\
\hline & $\mathrm{N}^{\circ}$ & $\%$ & $\mathrm{~N}^{\circ}$ & $\%$ & $\mathrm{~N}^{\circ}$ & $\%$ \\
\hline SI & 12 & 86 & 36 & 72 & 19 & 63 \\
\hline NO & 2 & 14 & 14 & 28 & 11 & 37 \\
\hline TOTAL & 14 & 100 & 50 & 100 & 30 & 100 \\
\hline \multirow{3}{*}{$\begin{array}{l}\text { En la mayoría de los casos de petición existe falta de } \\
\text { respuesta expresa a los pedidos formulados }\end{array}$} & \multicolumn{6}{|c|}{ Muestra } \\
\hline & \multicolumn{2}{|c|}{ Magistrados } & \multicolumn{2}{|c|}{ Abogados } & \multicolumn{2}{|c|}{ Docentes } \\
\hline & $\mathrm{N}^{\circ}$ & $\%$ & $\mathrm{~N}^{\circ}$ & $\%$ & $\mathrm{~N}^{\circ}$ & $\%$ \\
\hline SI & 12 & 86 & 34 & 68 & 24 & 80 \\
\hline NO & 2 & 14 & 16 & 32 & 6 & 20 \\
\hline TOTAL & 14 & 100 & 50 & 100 & 30 & 100 \\
\hline \multirow{3}{*}{$\begin{array}{l}\text { La entidad pública cumple con atender dentro del plazo legal } \\
\text { los pedidos formulados }\end{array}$} & \multicolumn{6}{|c|}{ Muestra } \\
\hline & \multicolumn{2}{|c|}{ Magistrados } & \multicolumn{2}{|c|}{ Abogados } & \multicolumn{2}{|c|}{ Docentes } \\
\hline & $\mathrm{N}^{\circ}$ & $\%$ & $\mathrm{~N}^{\circ}$ & $\%$ & $\mathrm{~N}^{\circ}$ & $\%$ \\
\hline SI & 0 & 0 & 8 & 16 & 4 & 13 \\
\hline
\end{tabular}




\begin{tabular}{|c|c|c|c|c|c|c|}
\hline NO & 14 & 100 & 42 & 84 & 26 & 87 \\
\hline TOTAL & 14 & 100 & 50 & 100 & 30 & 100 \\
\hline \multirow[t]{3}{*}{ Existe omisión de dar respuesta por escrito en el plazo legal } & \multicolumn{6}{|c|}{ Muestra } \\
\hline & \multicolumn{2}{|c|}{ Magistrados } & \multicolumn{2}{|c|}{ Abogados } & \multicolumn{2}{|c|}{ Docentes } \\
\hline & $\mathrm{N}^{\circ}$ & $\%$ & $\mathrm{~N}^{\circ}$ & $\%$ & $\mathrm{~N}^{\circ}$ & $\%$ \\
\hline SI & 13 & 93 & 40 & 80 & 26 & 87 \\
\hline NO & 1 & 7 & 10 & 20 & 4 & 13 \\
\hline TOTAL & 14 & 100 & 50 & 100 & 30 & 100 \\
\hline \multirow{3}{*}{$\begin{array}{l}\text { Omisión de dar respuesta por escrito en el plazo legal por la } \\
\text { entidad obligada }\end{array}$} & \multicolumn{6}{|c|}{ Muestra } \\
\hline & \multicolumn{2}{|c|}{ Magistrados } & \multicolumn{2}{|c|}{ Abogados } & \multicolumn{2}{|c|}{ Docentes } \\
\hline & $\mathrm{N}^{\circ}$ & $\%$ & $\mathrm{~N}^{\circ}$ & $\%$ & $\mathrm{~N}^{\circ}$ & $\%$ \\
\hline SI & 14 & 100 & 43 & 86 & 25 & 83 \\
\hline NO & 0 & 0 & 7 & 14 & 5 & 17 \\
\hline TOTAL & 14 & 100 & 50 & 100 & 30 & 100 \\
\hline \multirow{3}{*}{$\begin{array}{l}\text { La exigencia de procedimientos, requisitos y cobros no } \\
\text { contemplados en el TUPA de la entidad o norma legal, } \\
\text { originan las barreras burocráticas ilegales en las actuaciones } \\
\text { de los servidores públicos responsables. }\end{array}$} & \multicolumn{6}{|c|}{ Muestra } \\
\hline & \multicolumn{2}{|c|}{ Magistrados } & \multicolumn{2}{|c|}{ Abogados } & \multicolumn{2}{|c|}{ Docentes } \\
\hline & $\mathrm{N}^{\circ}$ & $\%$ & $\mathrm{~N}^{\circ}$ & $\%$ & $\mathrm{~N}^{\circ}$ & $\%$ \\
\hline $\mathrm{SI}$ & 13 & 93 & 40 & 80 & 25 & 83 \\
\hline NO & 1 & 7 & 10 & 20 & 5 & 17 \\
\hline TOTAL & 14 & 100 & 50 & 100 & 30 & 100 \\
\hline \multirow{3}{*}{$\begin{array}{l}\text { Uno de los factores que inciden en la vulneración al Derecho } \\
\text { de Petición son las barreras burocráticas ilegales al exigirse } \\
\text { procedimientos, requisitos y cobros no contemplados en el } \\
\text { TUPA de la entidad o norma legal. }\end{array}$} & \multicolumn{6}{|c|}{ Muestra } \\
\hline & \multicolumn{2}{|c|}{ Magistrados } & \multicolumn{2}{|c|}{ Abogados } & \multicolumn{2}{|c|}{ Docentes } \\
\hline & $\mathrm{N}^{\circ}$ & $\%$ & $\mathrm{~N}^{\circ}$ & $\%$ & $\mathrm{~N}^{\circ}$ & $\%$ \\
\hline SI & 13 & 93 & 38 & 76 & 26 & 87 \\
\hline NO & 1 & 7 & 12 & 24 & 4 & 13 \\
\hline TOTAL & 14 & 100 & 50 & 100 & 30 & 100 \\
\hline \multirow{3}{*}{$\begin{array}{l}\text { Las autoridades cumplen con fiscalizar la legalidad del } \\
\text { procedimiento administrativo en la petición. }\end{array}$} & & & Mu & & & \\
\hline & Magist & dos & Aboga & & Docen & \\
\hline & $\mathrm{N}^{\circ}$ & $\%$ & $\mathrm{~N}^{\circ}$ & $\%$ & $\mathrm{~N}^{\circ}$ & $\%$ \\
\hline SI & 1 & 7 & 13 & 26 & 5 & 17 \\
\hline NO & 13 & 93 & 37 & 74 & 25 & 83 \\
\hline TOTAL & 14 & 100 & 50 & 100 & 30 & 100 \\
\hline En la mayoría de casos presentados para la petición existe & & & $\mathrm{Mu}$ & & & \\
\hline debilidad en las labores de fiscalización por las autoridades & Magist & dos & Aboga & & Docen & \\
\hline encargadas & $\mathrm{N}^{\circ}$ & $\%$ & $\mathrm{~N}^{\circ}$ & $\%$ & $\mathrm{~N}^{\circ}$ & $\%$ \\
\hline SI & 14 & 100 & 40 & 80 & 26 & 87 \\
\hline NO & 0 & 0 & 10 & 20 & 4 & 13 \\
\hline TOTAL & 14 & 100 & 50 & 100 & 30 & 100 \\
\hline En la mayoría de casos presentados para la petición existe & & & $\mathrm{Mu}$ & & & \\
\hline vulneración al Derecho de Petición. & Magist & dos & Aboga & & Docen & \\
\hline & $\mathrm{N}^{\circ}$ & $\%$ & $\mathrm{~N}^{\circ}$ & $\%$ & $\mathrm{~N}^{\circ}$ & $\%$ \\
\hline SI & 13 & 93 & 40 & 80 & 26 & 87 \\
\hline NO & 1 & 7 & 10 & 20 & 4 & 13 \\
\hline TOTAL & 14 & 100 & 50 & 100 & 30 & 100 \\
\hline Las autoridades pertinentes deben implementar mecanismos & & & $\mathrm{Mu}$ & & & \\
\hline legales y administrativos para hacer efectivo el Derecho de & Magist & dos & Aboga & & Docent & \\
\hline $\begin{array}{l}\text { Petición el cual constituye un derecho fundamental a } \\
\text { formular peticiones y obtener una respuesta expresa dentro } \\
\text { del plazo legal. }\end{array}$ & $\mathrm{N}^{\circ}$ & $\%$ & $\mathrm{~N}^{\circ}$ & $\%$ & $\mathrm{~N}^{\circ}$ & $\%$ \\
\hline SI & 14 & 100 & 41 & 82 & 28 & 93 \\
\hline NO & 0 & 0 & 9 & 18 & 2 & 7 \\
\hline TOTAL & 14 & 100 & 50 & 100 & 30 & 100 \\
\hline
\end{tabular}

El $100 \%$ de los Magistrados, el $90 \%$ de los Abogados y $90 \%$ de los Docentes encuestados señalan que, si tienen conocimiento sobre la petición que comporta un conjunto de 
obligaciones explícitas o implícitas para la autoridad, en el que se incluye el derecho a obtener una respuesta por escrito dentro del plazo legal, bajo responsabilidad.

El $86 \%$ de los Magistrados, El $72 \%$ de los Abogados y $63 \%$ de los Docentes señalan que en la mayoría de los casos de petición existe apatía de las autoridades al resolver los pedidos sin observar el plazo legal; $y$, el $2 \%$ señala lo contrario.

El $86 \%$ de los Magistrados, $68 \%$ de los Abogados y $80 \%$ de los Docentes señalan que en la mayoría de los casos de petición existe falta de respuesta expresa a los pedidos formulados; $y$, el $14 \%$ señala lo contrario.

El $100 \%$ de los Magistrados, $84 \%$ de los Abogados y $87 \%$ de los Docentes señalan que en la mayoría de los casos de petición la entidad pública "no" cumple con atender dentro del plazo legal los pedidos formulados.

El $93 \%$ de los Magistrados, $80 \%$ de los Abogados y $87 \%$ de los Docentes señalan que en la mayoría de los casos de petición existe omisión de dar respuesta por escrito en el plazo legal por la entidad obligada; $y$, el $7 \%$ señala lo contrario.

El $100 \%$ de los Magistrados, $86 \%$ de los Abogados y $83 \%$ de los Docentes señalan que uno de los factores que inciden en la vulneración al Derecho de Petición es la omisión de dar respuesta por escrito en el plazo legal por la entidad obligada.

El $93 \%$ de los Magistrados, $80 \%$ de los Abogados y $83 \%$ de los Docentes señalan que en la mayoría de los casos de petición la exigencia de procedimientos, requisitos y cobros no contemplados en el TUPA de la entidad o norma legal, originan las barreras burocráticas ilegales en las actuaciones de los servidores públicos responsables; $y$, el 7\% señala lo contrario.

El $93 \%$ de los Magistrados, $76 \%$ de los Abogados y $87 \%$ de los Docentes señalan que uno de los factores que inciden en la vulneración al Derecho de Petición son las barreras burocráticas ilegales al exigirse procedimientos, requisitos y cobros no contemplados en el TUPA de la entidad o norma legal; $y$, el $7 \%$ señala lo contrario.

El 93\% de los Magistrados, $74 \%$ de los Abogados y $83 \%$ de los Docentes señalan que las autoridades "no" cumplen con fiscalizar la legalidad del procedimiento administrativo en la petición; $y$, el $7 \%$ señala lo contrario.

El $100 \%$ de los Magistrados, $80 \%$ de los Abogados y $87 \%$ de los Docentes señalan que en la mayoría de casos presentados para la petición existe debilidad en las labores de fiscalización por las autoridades encargadas.

El 93\% de los Magistrados, $80 \%$ de los Abogados y $87 \%$ de los Docentes señalan que en la mayoría de casos presentados para la petición existe vulneración al Derecho de Petición; y, el 7\% señala lo contrario.

El $100 \%$ de los Magistrados, $82 \%$ de los Abogados y $93 \%$ de los Docentes encuestados señalan que las autoridades pertinentes deben implementar mecanismos legales y administrativos para hacer efectivo el Derecho de Petición el cual constituye un derecho fundamental a formular peticiones y obtener una respuesta expresa dentro del plazo legal.

En la tabla 2, se presentan los casos sobre quejas sobre el Derecho de Petición, en la Defensoría del Pueblo - OD Tacna y de acuerdo a su Sistema de Información Defensorial (SID) se han presentado 111 quejas, dirigidas por ciudadanos (as) en contra de las Entidades Públicas de la región de Tacna; desde el 01.01.2016 al 31.12.2016. Es de 
resaltar que el $94 \%$ de los casos son relacionados a la omisión de dar respuesta por escrito en el plazo legal.

Tabla 2

Quejas sobre vulneración al Derecho de Petición, en la Defensoría del Pueblo - OD Tacna. Periodo 2016.

\begin{tabular}{clcc}
\hline $\mathrm{N}^{\circ}$ & \multicolumn{1}{c}{ Descripción } & Frecuencia & $\%$ \\
\hline 1 & Omisión de dar respuesta por escrito en el plazo legal. & 104 & $94 \%$ \\
2 & Respuesta no motivada, parcial o incongruente. & 3 & $3 \%$ \\
3 & Negativa a recibir peticiones. & 4 & $4 \%$ \\
& & 111 & $100 \%$ \\
\hline
\end{tabular}

Fuente: Defensoría del Pueblo - OD Tacna

En la tabla 3, se presentan los casos sobre quejas por omisión de dar respuesta por escrito en el plazo legal, de la Defensoría del Pueblo, siendo 104 quejas por omisión, de las cuales en el $89 \%$ se acreditó la vulneración del derecho y por ende el plazo legal.

Tabla 3

Quejas por omisión de dar respuesta por escrito en el plazo legal, en la Defensoría del Pueblo - OD Tacna. Periodo 2016.

\begin{tabular}{clcc}
\hline $\mathbf{N}^{\circ}$ & \multicolumn{1}{c}{ Descripción } & Frecuencia & $\mathbf{\%}$ \\
\hline $\mathbf{1}$ & $\begin{array}{l}\text { Quejas Fundadas (significa que se acreditó la vulneración del } \\
\text { derecho). } \\
\text { Quejas Infundadas (significa que no se acreditó la vulneración del } \\
\text { derecho). }\end{array}$ & $\mathbf{9 3}$ & $\mathbf{8 9 \%}$ \\
$\mathbf{3}$ & $\begin{array}{l}\text { Quejas Inconclusas (significa que no fue posible determinar la } \\
\text { vulneración o no del derecho, por falta de respuesta de la entidad). } \\
\text { TOTAL }\end{array}$ & $\mathbf{3 \%}$ \\
\hline
\end{tabular}

Fuente: Defensoría del Pueblo - OD Tacna

En la tabla 4, se presentan las cinco instituciones más quejadas de la región de Tacna, debido a la omisión de dar respuesta por escrito en el plazo legal de la Defensoría del Pueblo. La Municipalidad Provincial de Tacna es la institución más quejada por vulneración al Derecho de Petición.

Tabla 4

Las cinco instituciones más quejadas por omisión de dar respuesta por escrito en el plazo legal, en la Defensoría del Pueblo - OD Tacna. Periodo 2016.

\begin{tabular}{clcc}
\hline $\mathbf{N}^{\circ}$ & \multicolumn{1}{c}{ Institución } & Frecuencia & $\mathbf{\%}$ \\
\hline $\mathbf{1}$ & Municipalidad Provincial de Tacna & 22 & $21 \%$ \\
$\mathbf{2}$ & Gobierno Regional de Tacna & 18 & $17 \%$ \\
& Municipalidad Distrital del Alto de la Alianza & 10 & $10 \%$ \\
$\mathbf{3}$ & Unidades de Gestión Educativa Local (Ugel) & 8 & $8 \%$ \\
$\mathbf{4}$ & Municipalidad Distrital de Coronel Gregorio Albarracín & 6 & $6 \%$ \\
$\mathbf{6}$ & Otros & 40 & $38 \%$ \\
TOTAL & $\mathbf{1 0 4}$ & $\mathbf{1 0 0 \%}$
\end{tabular}

Fuente: Defensoría del Pueblo - OD Tacna 


\section{Resultados de la entrevista a Magistrados:}

1. ¿Considera usted que uno de los factores que inciden en la vulneración al Derecho de Petición es la omisión de dar respuesta por escrito en el plazo legal por la entidad obligada? Fundamente.

Sí, en efecto son cotidianos y múltiples los ejemplos en los que se observa una demora excesiva en la resolución de las peticiones formuladas por los ciudadanos; debido a que los servidores públicos y funcionarios incurren en omisiones derivadas del incumplimiento de plazos.

2. ¿Considera usted que en la mayoría de los casos de petición la exigencia de procedimientos, requisitos y cobros no contemplados en el TUPA de la entidad o norma legal, originan las barreras burocráticas ilegales en las actuaciones de los servidores públicos responsables? Fundamente.

Sí, ya que el Derecho de Petición es un derecho fundamental, por lo que no debe estar supeditado a ningún cobro y/o exigencia no establecida en el TUPA de la entidad. Al respecto, considero que la existencia de trámites complejos y carentes de transparencia en los que se advierte una inadecuada preparación del servidor público.

3. ¿Considera usted que uno de los factores que inciden en la vulneración al Derecho de Petición son las barreras burocráticas ilegales al exigirse procedimientos, requisitos y cobros no contemplados en el TUPA de la entidad o norma legal? Fundamente.

Sí, esta situación acontece cuando se fijan los derechos por los servicios administrativos que prestan, las entidades de alcance nacional, regional y local, la cual no publican oportunamente sus TUPAs y modificaciones en sus portales institucionales, como también un ejemplar en físico a la vista del ciudadano.

4. ¿Considera usted que en la mayoría de casos presentados para la petición existe debilidad en las labores de fiscalización por las autoridades encargadas?

Sí, ello se debe a que las autoridades encargadas no realizan una labor permanente de supervisión y seguimiento de los casos de petición. Máxime, se advierte que las entidades públicas no fortalecen sus oficinas de control para realizar labores de fiscalización en el tema. Asimismo, es notoria la falta de interés por parte del Estado para la atención de este derecho.

5. ¿Considera usted que en la mayoría de casos presentados para la petición existe vulneración al Derecho de Petición?

Sí, vemos de manera frecuente que los servidores públicos y/o autoridades no atienden dentro del plazo legal las peticiones de los ciudadanos. A su vez que, aplican requisitos y procedimientos no contemplados en el TUPA de la entidad.

6. ¿Qué mecanismos legales y administrativos se pueden implementar para poder garantizar el Derecho de Petición? Fundamente.

Se requiriere un control y seguimiento permanente de las peticiones que se formulen; así como sanciones drásticas a los servidores públicos y/o autoridades encargados, que omitan sus funciones. Para cuyo efecto podría crearse una entidad autónoma en específico que asuma esta función y tenga capacidad vinculante o coercitiva para sus decisiones. Tenemos si bien cierto a la Defensoría del Pueblo y al INDECOPI; empero no es suficiente ya que, como mencioné se requiere de una entidad especializada en el tema.

\section{DISCUSIÓN}

La presente investigación determinó los principales factores que están incidiendo en la vulneración al Derecho de Petición; ya que se comprobó que: La omisión de dar respuesta por 
escrito en el plazo legal, las barreras burocráticas ilegales por la entidad obligada, y la debilidad de las labores de fiscalización por las autoridades son los principales factores que inciden en la vulneración al Derecho de Petición. Tacna, 2016. En la mayoría de casos presentados para la petición la entidad obligada omite dar respuesta por escrito en el plazo legal, al hallarse que existe apatía, falta de respuesta expresa y en la mayoría de los casos de petición existe omisión de dar respuesta por escrito en el plazo legal por la entidad obligada de las autoridades al resolver los pedidos sin observar el plazo legal. Se dan las barreras burocráticas ilegales, al exigirse procedimientos, requisitos y/o cobros no contemplados en el TUPA de la entidad o norma legal con debilidad de las labores de fiscalización de las autoridades encargadas. Las autoridades "no" cumplen con fiscalizar la legalidad del procedimiento administrativo en la petición. Es importante resaltar que la petición es un derecho fundamental y es uno de los derechos más ejercidos por la ciudadanía, ya que posibilita la comunicación directa con el Estado. En efecto, son cotidianos y múltiples los casos en los que se observa una demora excesiva en la resolución de las peticiones formuladas por los ciudadanos (as). Por lo que, superar esta deficiencia constituye uno de los mayores desafíos que tiene el proceso de reforma y modernización del Estado. La Presidencia del Consejo de Ministros, a través de la Secretaría de Gestión Pública debe asesorar a las entidades públicas en materia de simplificación, eficacia y calidad administrativa. Así también, la PCM y el Ministerio de Economía y Finanzas deben expedir el Decreto Supremo referida a la aprobación de los derechos de tramitación para los procedimientos estandarizados; en cumplimiento de lo dispuesto por el artículo $51^{\circ} .7$ de la Ley de Procedimiento Administrativo General. Los Órganos de Control Institucional (OCI) de cada entidad conforme a sus funciones deben supervisar permanentemente el cumplimiento de plazos, requisitos y procedimientos de acuerdo al TUPA de la entidad. Asimismo, elevar al titular de la entidad un informe mensual sobre el estado de las peticiones y los procedimientos administrativos. El Estado debe fortalecer e implementar el uso de las Tecnologías de la Información y Comunicación (TICS) en la administración pública, por cuanto facilitará la tramitación de numerosos procedimientos y permitirá de forma puntual conocer en cada momento el estado de las peticiones, así como solventar las dudas que puedan tener los interesados. Crear una entidad autónoma que de manera específica realice labores de fiscalización en el tema y tenga capacidad vinculante o coercitiva para sus decisiones. Además, que reciba, oriente, analice y cumpla con monitorear cada una de las peticiones que requieren los ciudadanos. Aprobar una norma que regule de manera específica el Derecho de Petición, al igual como la Ley N² 27806 "Ley de Transparencia y Acceso a la Información Pública”. Por cuanto, debe considerarse nuestro contexto jurídico-social y la experiencia del Estado Colombiano en este tema mediante Ley 1755 de 2015 y brindar capacitación permanente al personal de las dependencias, unidades o direcciones que tramitan procedimientos administrativos, sobre todo en las Municipalidades Provinciales y Distritales; considerando el alto nivel de quejas. Asimismo, el Estado en su conjunto debe realizar actividades informativas que permitan a los ciudadanos y ciudadanas conocer sus derechos y las posibilidades de intervención y control de las instituciones públicas. Las diversas entidades públicas deben fortalecer la efectividad de la Queja, a efectos que las quejas y denuncias presentadas por la ciudadanía permitan de manera efectiva prevenir y sancionar conductas irregulares de los servidores públicos en la administración pública, así como mejorar los trámites y servicios públicos que dan origen a dichas quejas. Promover la cultura de la buena administración pública y la satisfacción adecuada del derecho fundamental de petición, reconocido por nuestra Constitución Política. Publicar oportunamente sus TUPAs y modificaciones en sus portales institucionales, así como en físico. Abstenerse de exigir requisitos y cobros no contemplados en sus TUPAs o norma legal y procurar remover de oficio los obstáculos puramente formales y evitar las dilaciones y los retardos en las peticiones; a efectos de buscar la compatibilidad con la equidad y el servicio objetivo al interés general. 


\section{REFERENCIAS BIBLIOGRÁFICAS}

Aguilar Villanueva, L. F. (2008). Gobernanza y Gestión Pública. Fondo de Cultura Económica, Segunda reimpresión, México.

Badeni, G. (1995). Nuevos Derechos y Garantías Constitucionales. Editorial Ad-Hoc. Buenos Aires-Argentina.

Bartra Cavero, J. (2012). El Derecho de Petición. Artículo Jurídico. Lima-Perú. Recuperado de

Becerra Pinilla, J. (1995). El Derecho de Petición. Primera Edición. Bogotá Colombia: Ediciones Jurídicas Gustavo Ibáñez.

Berríos Llanco, E. (2012). La Obligación de la Administración Pública de brindar un Servicio de Calidad y el Derecho de todas las Personas a una Buena Administración. Gaceta Jurídica. Diálogo con la Jurisprudencia: actualidad, análisis y crítica jurisprudencial. 1a ed. Lima.

Bulla Romero, J. E. (2010). Derecho de Petición. 1a ed., Bogotá: Ediciones Nueva Jurídica.

Cabanellas, G. (1998). Diccionario de Derecho Usual, Vigésimo Sexta Edición. Tomo III, Editorial HELIASTA. Buenos Aires - Argentina.

Camarasa Casterá, J. J. (2004). La Calidad en la Administración Pública. En: Revista "Educar en el 2000", Murcia, abril.

Carrillo Artiles, C. L. (2001). El Derecho de Petición y la Oportuna y Adecuada Respuesta en la Constitución de 1999. Libro Homenaje a la Universidad Central de Venezuela. Edición conjunta del Tribunal Supremo de Justicia y la Universidad Central de Venezuela Caracas.

Chacón Molina, M. J. (2015). El Derecho de Petición y el Silencio. Monografía previa a la obtención del Título de Abogada de los Tribunales de Justicia de la Republica y Licenciada en Ciencias Políticas y Sociales. Universidad de Cuenca - Ecuador. Recuperado de

Cienfuegos Salgado, D. (2004). El Derecho de Petición en México. Instituto de Investigaciones Jurídicas de la Universidad Nacional Autónoma de México, 1era Edición - México, D.F.

Cordero Ordóñez, P. (2009). El Silencio Administrativo. Quito, Editorial El Conejo.

Defensoría del Pueblo (1998). Manual de Derechos Humanos, Administración Estatal y Servicios Públicos. Comisión Andina de Juristas.

Defensoría del Pueblo (2008). Informe Defensorial № 133. ¿Uso o abuso de la autonomía municipal? El desafío del desarrollo local. Elaborado por el Programa de Descentralización y Buen Gobierno de la Adjuntía para la Administración Estatal. 1era. Edición: Lima - Perú.

Defensoría del Pueblo (2009). Informe Defensorial № 145. Aplicación del Silencio Administrativo: Retos y tareas pendientes. Primera reimpresión: Lima-Perú.

Defensoría del Pueblo (2015). Informe Defensorial № 165. Balance a diez años de vigencia de la Ley de Transparencia y Acceso a la Información Pública 2003-2013. Elaborado por la Adjuntía en Asuntos Constitucionales de la Defensoría del Pueblo. Primera Edición: Lima - Perú.

Defensoría del Pueblo (2016). Vigésimo Informe Anual 2016. Primera Edición: Lima - Perú, mayo del 2017.

Del Castillo, R. (1976). Diccionario de Ciencias Sociales. Vol. II. Madrid: Instituto de Estudios Políticos.

Díaz Mendoza, M. A. (2013). El derecho de petición en la República Bolivariana de Venezuela. Caracas - Venezuela.

Díaz, A. A. (2008). La Calidad en la Gestión Pública. Programa conjunto de investigación CERIUNP, Paraguay.

Diez, M. M. (1974). Derecho Administrativo. Tomo II. Buenos Aires. 
Duguit, L. traducido por Acuña José G. (1926). Manual de Derecho Constitucional. Segunda Edición. Madrid-España: Editorial Librería Española y Extranjera Príncipe.

Ferrer Mac-Gregor Poisot, E; Caballero Ochoa, J. L; Steiner, C. (2013). Derechos Humanos en la Constitución: Comentarios de Jurisprudencia Constitucional e Interamericana I. Primera Edición - México, D.F

Franco Hermida, W. J. (2011). El Derecho de Petición frente al Nuevo Código Contencioso Administrativo. Trabajo de investigación presentado como requisito parcial para optar al título de: Magister en Derecho Administrativo. Universidad Nacional de Colombia Facultad de Derecho Ciencias Políticas y Sociales Bogotá, Colombia.

Gamboa Martínez, Y. V. (2014). Naturaleza Jurídica de la respuesta del Derecho de Petición. Universidad Militar Nueva Granada Facultad de Derecho Bogotá, Colombia.

García De Enterría, E. (2006). Curso de Derecho Administrativo. Tomo II. España.

Garrido Falla, F. (1955). La llamada doctrina del silencio administrativo. Revista de Administración Pública № 16. Madrid: Instituto de Estudios Políticos, Enero / Abril.

Gutiérrez, W. (2005). La Constitución comentada Tomo I: Análisis artículo por artículo. Gaceta Jurídica Congreso de La Republica del Perú. Primera Edición. Lima - Perú.

Indecopi (2017). Manual sobre Prevención y Eliminación de Barreras Burocráticas.

Luna Cervantes, E. (2005). En comentario al Artículo $2^{\circ}$ Inciso 20 de la Constitución Política del Perú de 1993. "La Constitución Comentada: Análisis de artículo por artículo". Tomo I. Edit. Gaceta Jurídica. Lima-Perú.

Martínez Ceballos, G. J. (2002). El Derecho de Petición, Primera Edición. Bogotá - Colombia.

Morón Urbina, J. C. (2008). Comentarios a la Ley del Procedimiento Administrativo General. 7 ma. edición. Lima: Gaceta Jurídica S.A.

Morón Urbina, J. C. (2011). Comentarios a la Ley del Procedimiento Administrativo General. Lima-Perú, 9a Ed. Gaceta Jurídica.

Olano, C. A. (1987). Derecho Constitucional e Instituciones Políticas. Editorial Temis. Segunda Edición. Bogotá - Colombia.

Pérez Escobar, J. (1997). Derecho Constitucional Colombiano. TEMIS. Bogotá - Colombia.

Pérez Royo, J. (2000). Curso de Derecho Constitucional. Séptima Edición. Marcial Pons, Ediciones Jurídicas y Sociales S.A., Madrid-España. Recuperado de

Sachica, L. C. (1966). Constitucionalismo Colombiano. Editorial Temis. Segunda Edición. BogotáColombia.

Salazar Chávez, R. (1999). El Derecho de Petición y la Administración Pública en el Perú. THEMIS 39, Lima - Perú. Recuperado de http://dialnet.unirioja.es/descarga/articulo/5110174.pdf

Santofimio Gamboa, J. (2004). Resolución de la Petición: Tratado de derecho administrativo. Tomo II. 4ạedición.

Sar Suarez, O. (2012). Constitución Política del Perú de 1993. Sumillada, Concordada y Anotada artículo por artículo, con los precedentes y jurisprudencia vinculante del Tribunal Constitucional. Investigación realizada por el Centro de Estudios de Derecho Constitucional. Lima-Perú.

Uribe Giraldo, L. E. (1984). El Estado, las libertades individuales y el preámbulo de la constitución. Pontificia Universidad Javeriana. Bogotá-Colombia.

Vidal Vergara, C. (1936). Los derechos individuales en las constituciones modernas. Editorial Nascimiento. Santiago de Chile-Chile.

Wolf, E. (1945). Tratado de Derecho Constitucional Venezolano. Tipografía Americana. Caracas - Venezuela. Tomo II. 